\title{
Platformation: Cloud Computing Tools at the Service of Social Change
}

\author{
Anil Patel
}

\author{
(6) 18 \\ Because when ideas are shared, the possibilities don't add up. \\ They multiply. \\ Paul Romer \\ Economist, Entrepreneur, and Activist
}

\begin{abstract}
The following article establishes some context and definitions for what is termed the "sharing imperative" - a movement or tendency towards sharing information online and in real time that has rapidly transformed several industries. As internet-enabled devices proliferate to all corners of the globe, ways of working and accessing information have changed. Users now expect to be able to access the products, services, and information that they want from anywhere, at any time, on any device.
\end{abstract}

This article addresses how the nonprofit sector might respond to those demands by embracing the sharing imperative. It suggests that how well an organization shares has become one of the most pressing governance questions a nonprofit organization must tackle. Finally, the article introduces Platformation, a project whereby tools that enable better inter and intra-organizational sharing are tested for scalability, affordability, interoperability, and security, all with a non-profit lens.

\section{Introduction}

There are now more devices connected to the Internet than there are people on the planet (tinyurl.com/3rk9yev). By 2020, CISCO predicts that 50 billion devices will be connected (tinyurl.com/7flbk3k). While some may feel overwhelmed by this proliferation of connectedness, others see opportunity. We envision connectivity as a driver of social change.

The implications of connectivity on business have been profound, as we will discuss later in this article. The same could be true for social change, but some "big picture" thinking will have to take place if this sector is to become equally adept at leveraging new ways of working and learning.

What would be the result if Canada's small percentage of the global do-good community were interconnected - if curated pieces of financial, operational, and programmatic information were connected online and in real-time for staff, volunteers, donors, and other likeminded organizations to engage with and share? Consider one small, practical example: if just $10 \%$ of these organizations agreed to share a budget document that reflected each organization's anticipated printing needs over the next 12 months. One possible outcome of this sharing might be a commercial printer lobbying for their business because it represents much more than a few piecemeal jobs scattered throughout the year; this shared budget represents a larger win for the printer. The power of nonprofits to leverage their spending power for competitive rates is largely unexplored. However, a second potential outcome is far more interesting. If these organizations agree to retain a social enterprise printer, this scenario allows the social enterprise printer to secure predictable business, build capacity, and increase impact.

Sharing just one small piece of information - such as a printing schedule - would also make it easier to understand what like-minded organizations are doing right 


\section{Platformation: Cloud Computing Tools at the Service of Social Change}

Anil Patel

now and what they are doing in the future as well. This is important because the public and donor-based pressures being exerted upon nonprofits revolve around collaboration, finding efficiencies, and saving costs.

Getting better at sharing key information is also of critical importance to the Canadians who collectively contribute 160 million volunteer hours in a governance-related capacity, which includes sitting on boards, participating in finance committees, and chairing fundraising committees (tinyurl.com/84bwh3m). For each of these volunteers, their key activities revolve around reviewing, commenting upon, and in some cases, disseminating mission-critical pieces of information. When these volunteers are armed with key pieces of information - in real time and accessible from anywhere - they will be empowered to be better, more effective volunteers.

Social media has taught us that providing the right information at the right time will encourage greater engagement from stakeholders. For this reason, many "do-good" organizations seek to operate like the "open web", which refers to an open and standardized infrastructure underlying the Internet. As stated in the book, An Open Web (tinyurl.com/butfwqz), "it is this very openness that has allowed for an unprecedented level of innovation, knowledge generation and creative expression on the Web and off." The authors cited Wikipedia (wikipedia.org) as: "the canonical demonstration of openness. Its combined cognitive output, technically and normatively interoperable and infinitely modifiable, propelled it into one of the most well-known bodies of knowledge in human history."

A non-profit sector that could combine knowledge and resources in a similar way, while allowing for infinite modifications, and not being limited by proprietary interfaces or platforms, would be operating like the open web. However, not everything a nonprofit does can be shared with everyone all the time. The real challenge to "do-good" organizations that would like to operate like the open web is this: how to determine which pieces of data need to be completely private (e.g., social enterprise client contact information) versus completely public (e.g., number of clients served). Understanding what to share when, with whom, and how to share it means that an organization needs to put a strong information and communication technology plan into place. Governance rules and protocols are critical and essential parts of this plan.
This article summarizes how visionary applications of cloud computing have transformed many different industries, before asking how the same vision could also change philanthropy. The article reveals what is possible for social organizations "in the cloud" and explores the new skills that organizations must develop to navigate the simultaneous demands for privacy, transparency, effectiveness, and collaboration. It also provides concrete examples of new platforms for information sharing that would benefit organizations of all shapes and sizes to enable social change.

\section{Trends that Influence Technology Decisions}

In order to properly explore how cloud-computing tools can contribute to social change, we need to first understand the cultural and organizational trends that strongly influence technology decisions. The following emerging themes and questions shape our thinking in this article:

1. The sharing imperative: If other industries have undergone rapid and unprecedented change, what are the applicable trends that will also affect philanthropy?

2. Sharing as a governance question: If effective 21stcentury philanthropy requires new approaches to efficiency, collaboration, and transparency, what are the new tools and architecture to enable these approaches?

3. The art of the possible: If we are to re-envision what pieces of financial, operational, and programmatic information are shared online and in real time, what are some concrete examples that justify further inquiry and testing?

The pressure to be collaborative, transparent, and efficient Curating the right information to the right people becomes something around which all other governance principles are shaped. Get sharing right, and organizations will flourish in good times and bad. Get it wrong, and the results are donor fatigue and information overload, among other undesirable side effects, as the examples in this section will demonstrate.

Reduction in government grants is widely expected as governments fight debt and deficits (Perry, 2011; tinyurl .com/ccgjcvr). The February issue of Imagine Canada's Sector Monitor (Lasby and Barr, 2012; tinyurl.com/crqtnnl) indicated that one-quarter of respondents believe the future of their organization is at risk and two-fifths of leaders expect an increase in demand for their services. 


\section{Platformation: Cloud Computing Tools at the Service of Social Change}

\section{Anil Patel}

Reduced levels of funding in the face of increased demand for services are paradoxical and the situation is unsustainable. Additionally, donor fatigue and dissatisfaction continue to rise due to the number and nature of requests individuals are receiving (Turcotte, 2012; tinyurl.com/btvldcc). This puts even more pressure on already cash-strapped charities.

These factors, and others, contribute to concrete management challenges for nonprofit leadership. These management challenges stem from a deeply felt pressure to be collaborative, transparent, and efficient and not just to be good in one of these areas, but to enact all three simultaneously. Some examples of this pressure are outlined below.

In March 2012, the Drummond Report (tinyurl.com/ 8yvzycq) outlined 326 recommendations that the Government of Ontario could take to eliminate the deficit and reduce debt. The Ontario Nonprofit Network (tinyurl.com/ 7f7g2jw) analyzed the Drummond Report and documented key words and phrases that are relevant to the nonprofit sector, including:

- new approaches

- rethink accountability

- increase transparency

- explore further partnerships

In February 2011, the Canadian Broadcasting Corporation caught the Canadian Cancer Society flat footed when it aired a two-part investigative report scrutinizing 10 years of financial data and program results (tinyurl .com/44uxurz). Mark Blumberg, a tax lawyer who works with nonprofits and charities, was quoted in the article saying: "In the end, they need to listen to all the different interest groups and stakeholders that have an interest in the area, and just not prefer one and ignore the others."

In the global arena, the International Aid Transparency Initiative (aidtransparency.net/iati-standard) is putting new pressure on aid organizations of all shapes and sizes to demonstrate value for money. In Canada, Imagine Canada's Standards Initiative (imaginecanada.ca/standards _initiative) has just announced the first cohort of organizations to pass through their time-intensive and rigorous accreditation process, which was "designed to strengthen public confidence in the charitable and nonprofit sector".
These examples demonstrate the tremendous pressure exerted on nonprofits to demonstrate transparency, collaboration, and efficiency. If sharing is the way that efficiency, collaboration, and transparency can co-mingle, then we need to ask what steps need to be taken in order to put in place good IT systems and protocols around sharing.

\section{Building an IT system for sharing}

The sharing imperative teaches us that, for better or worse, the more people are connected to the Internet, the more they expect access to what they want, when they want it. As such, do-good organizations must be prepared to meet these new user expectations. One way to prepare is to encourage do-good organizations to make sharing information, online and in real time, a critical governance question. Now more than ever, an organization must have clear parameters, defined at the board level, that determine how, what, when, where, and with whom an organization shares information. Though it sounds as though these rules will constrain the flow of information, it is actually quite the opposite. Strong protocols mean that more people can be empowered to strategically deploy specific pieces of information to further organizational goals.

Figure 1 characterizes some of the thinking that a nonprofit must go through to build an IT system for sharing. An organization needs to take into account all of its financial, operational and programmatic information and decide what needs to be fully-private, semi-private, semi-public, or fully-public. As we move from the left to the right side of the figure, we move from hard data and numbers, through information and mechanics, and land on stories, effects, and meaning.

Consider how Figure 1 might be applied to the example of a small social service organization that has a few staff, several dozen dedicated volunteers, and annual revenue just over $\$ 1$ million:

1. Activities tend to be easy to predict. For instance, how many youth will be served at a local community centre for after-school programming they otherwise could not afford? What is the cost structure to provide adequate programming? What is the nature of the revenue sources? Does it cover the true cost of running the programming? If printing and catering are required, is there a local social enterprise that provides these services?

2. Outcomes are somewhat more predictable, but still generate some unknowns. For instance, of the particip- 


\section{Platformation: Cloud Computing Tools at the Service of Social Change}

\section{Anil Patel}

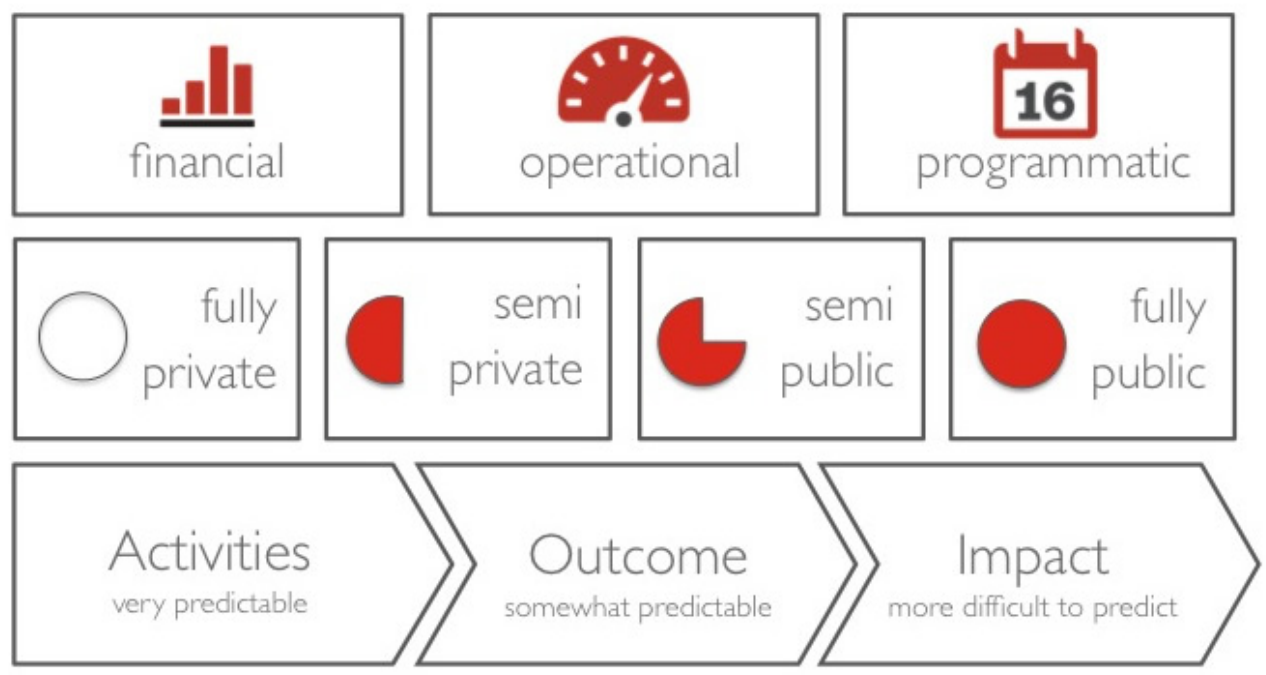

Figure 1. Learn to share what you need to share

ating youth, which ones felt the service was of value? For the ones who did, why? For those that did not, why not? Are staff and volunteers provided adequate training and supervision to ensure the programming is carried out?

3. Impact usually requires a much longer time period to generate results that can be analyzed. For instance, what is the true social return on investment that takes into account all the time and money needed? Has the appropriate amount of attribution been placed on the intervention? Compared to all the similar afterschool programming available to youth, how does this specific programming compare in terms of lasting change, scalability, or replication?

This type of thinking is essential for an organization to assess its information and consider how an IT system can address the pressures on collaboration, transparency, and efficiency. Once an organization has adopted a sharing mind-set, what IT tools are appropriate to put a system in place? In the next section, we introduce a project that is designed to help nonprofit organizations identify and use the tools they need to achieve their sharing goals.

\section{Platformation}

Thus far, the focus of this article has not been on technology, but rather it has been on the mind-set that a nonprofit organization needs to adopt (e.g., "we want to collaborate more", "we want to be more efficient", "we want to be more transparent"), and the realization that follows (e.g., "sharing will help us do all three"). But what are the tools that a nonprofit can use that will help them get better at sharing? What tools will allow an organization to curate information online and in real time, while being both cost effective and scalable. Platformation has sought to address these questions.

Platformation (platformation.ca) is an online resource for members of the charitable sector interested in implementing free and low-cost online and mobile technology into their IT infrastructure.

Platformation prototypes new platforms for information sharing (platform + information = Platformation), which means it tests technological approaches that will enable nonprofits to operate like the open web.

As part of Platformation, the team at Framework (frameworkorg.org) has been testing more than 200 cloudcomputing tools across a range of criteria including cost, security, customer service, scalability, and interoperability. We have looked at tools across 30 different categories, from accounting to project management to online ticket sales, to domain hosting. Coming as we do from the nonprofit sector, all tools are evaluated from this perspective.

One of the key criteria that we test for is interoperability; or, how well do tools (often from different software vendors) work with each other. We call this approach: "open architecture". Open architecture differs from open source in one fundamental way. Open architecture is a software procurement strategy that considers 


\section{Platformation: Cloud Computing Tools at the Service of Social Change}

\section{Anil Patel}

how well one software application provider "talks to" another, regardless of whether it is open source or proprietary. It also takes into consideration things such as, but not limited to, vendor viability (e.g., will they go bankrupt?), how securely they store customer data, customer support, and pricing plans. For instance, the filesharing provider Box.com (box.com) allows file sharing with project management provider SmartSheet (smartsheet.com), reducing the need to log in at two different places. Similarly, SmartSheet integrates with the Salesforce.com (salesforce.com) relationship management system, as does Box.com; all three are integrated with Google Apps for Business (google.com/enterprise/apps/ business/).

Furthermore, core pieces of data, information, and documents within can be selectively shared with different levels of permissions and visibility. In the case of Box.com, budgets can be securely shared with specific users or pre-approved web addresses or embedded on public web pages. SmartSheet project documents that are read-only, can also be shared via URLs (web addresses) or embedded on web pages. For less sensitive information, an embedded or shared SmartSheet can have viewable attachments and discussions. The combined power of these tools is impressive.

When evaluating the total cost of subscribing to these tools, organizations need to evaluate the sharing and collaborative features of the whole software-as-a-service stack (SaaS stack). For a team of five people, a budget between $\$ 1,000$ to $\$ 2,500$ per year would be needed to run the organization from the Internet:

- Salesforce: basic contact manager at $\$ 5 /$ user/month

- Box.com: \$15/user/month

- SmartSheet: \$500 for 150 sheets and 3 administrative users

- Google Apps for Business: \$50/user/year

In this particular configuration, users can decide where and how much information is shared with a variety of stakeholders.

By testing potential applications and forming a collaborative with other groups working at the intersection of technology and nonprofit organizations, Platformation provides nonprofit organizations with a menu of cloudcomputing options to suit their organization's sharing needs. Of course, before an organization can begin to choose tools that enable sharing, sharing must be addressed as a governance question, as we emphasized above. As Platformation becomes increasingly useful as a hardware store of sorts, where the browser can be sure all the tools on display are of the highest quality, so too must we be aware that tools are only as good as the plan that determines how they will be used.

As a number of organizations have begun to draw on the knowledge base that is Platformation, they have simultaneously had to spend some time thinking through their organization's relationship to technology. Is the responsibility for technology decisions in one person's hands, or distributed broadly? Does technology enable or impede mission success? Do our technology choices enable room for growth and innovation, or are we bound by the limits of the tools we have chosen?

All these, and more, are critical questions that must be posed by any organization as they construct their optimal platform to enable information sharing.

\section{Applying open architecture to nonprofit impact}

Consider the time that can be freed up by making basic financial information available online and in real time and making it available to vendors, volunteers, funders, and others, to access in their own time. As shown in Figure 2, imagine that Nonprofit A has begun sharing its 12-month printing budget forecast on its website. The budget spreadsheet and printing specs folder can be shared as easily as a Youtube video.

The manager at "Social Enterprise Printing" learns about the forecast document and then quickly pulls the data into its web infrastructure by archiving its URL or HTML code. The same budget spreadsheet and printing needs are now visible in two places. This signals another like-minded organization - Nonprofit B - to consider creating and sharing a similar forecast using a similar method on its own IT infrastructure, which the manager at Social Entreprise Printing also pulls into its architecture.

Next consider Funder A, who funds all three organizations and who can now instantly see the connections that are being made. Simultaneously, the information is shared with Funder B, who has been trying to establish new granting principles around impact investing, and now encourages Nonprofit $\mathrm{C}$ to consider doing the same.

Now imagine this sharing has happened amongst just $10 \%$ of the nonprofit community in Canada. With limited cost and technical know-how, an information superhighway that has improved the IT capacity of each participating organization, has also potentially influ- 


\section{Platformation: Cloud Computing Tools at the Service of Social Change}

\section{Anil Patel}

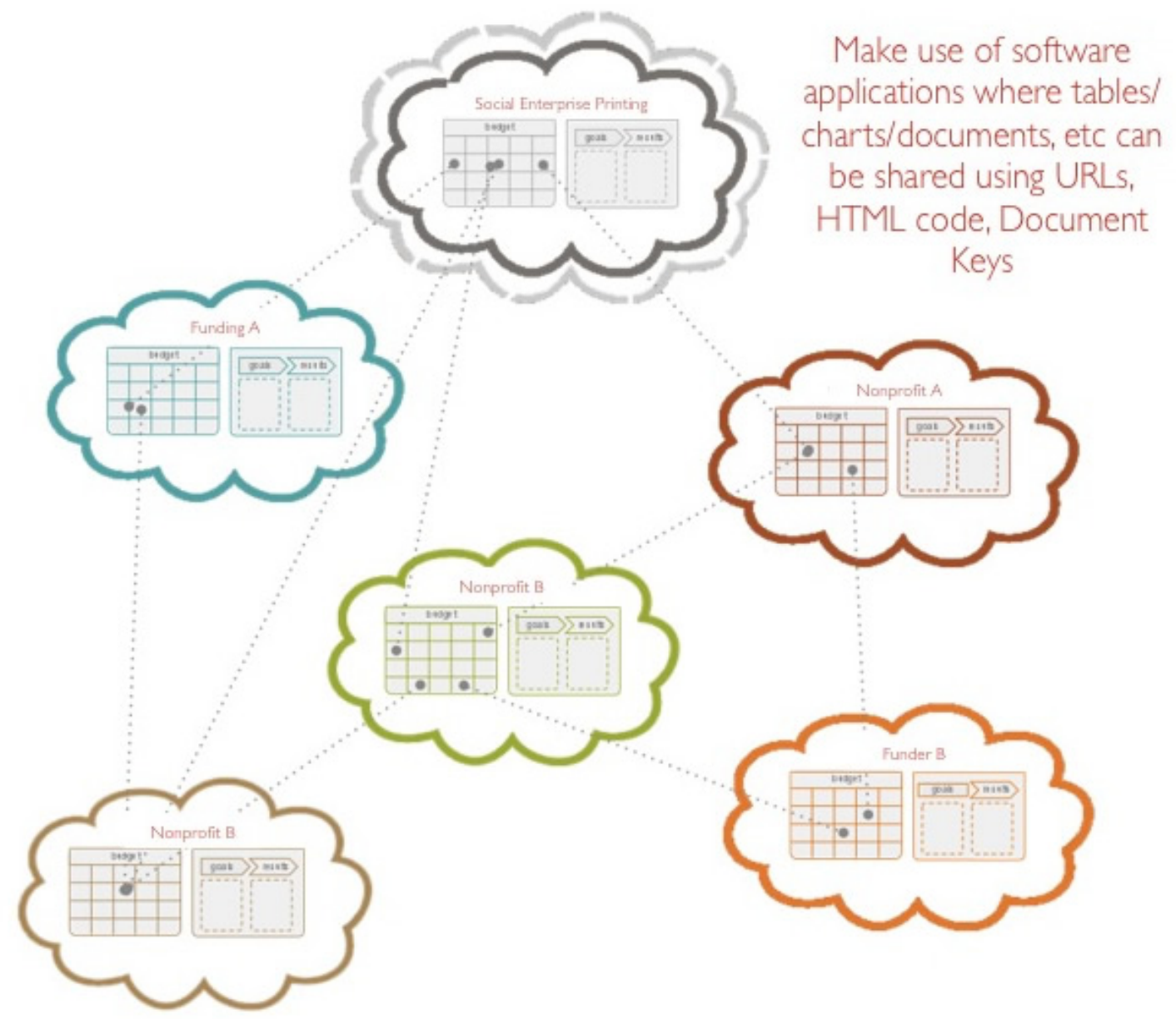

Figure 2. Allow information to flow freely

enced the way that millions of dollars flow between likeminded organizations, both nonprofit and social enterprise. This is the vision that Platformation is working to realize.

\section{Conclusion}

This article explored the trends that are driving the sharing imperative and asked how this imperative will affect the nonprofit sector. As more and more people are connected to the internet and demanding immediate access to information, the expectations and pressures on the nonprofit sector have changed. There are many benefits to sharing key pieces of financial, operational, and programmatic information; by testing cloudbased tools for their suitability for nonprofit organizational needs, Platformation is helping nonprofits realize these benefits through increased levels of collaboration, transparency, and efficiency.

\section{About the Author}

Anil Patel is an Ashoka Fellow and Executive Director of Framework, the charity that runs the Timeraiser. The Timeraiser is part volunteer fair, part silent art auction, and part night on the town. To date, the Timeraiser has generated 100,000 volunteer hours, engaged 6,500 Canadians to pick up a cause, worked with more than 350 agencies in need of skilled volunteers, and invested $\$ 580,000$ in the careers of Canadian artists. In the decade ahead, Anil will be focused on how nonprofits and funding organizations can share critical information online and in real time. 